08

\title{
Распределение намагниченности в частицах с конфигурационной анизотропией, полученных методом микросферной литографии
}

\author{
() Д.А. Бизяев, ${ }^{1}$ А.А. Бухараев, ${ }^{1}$ Н.И. Нургазизов, ${ }^{1}$ А.П. Чукланов, ${ }^{1}$ В.М. Масалов ${ }^{2}$ \\ ${ }^{1}$ Казанский фризико-технический институт им. Е.К. Завойского, Федеральный исследовательский центр „Казанский \\ научный центр РАH“, \\ 420029 Казань, Россия \\ ${ }^{2}$ Институт фоизики твердого тела РАН, \\ 142432 Черноголовка, Россия \\ e-mail: dbiziaev@inbox.ru
}

Поступило в Редакцию 28 марта 2019 г.

В окончательной редакции 28 марта 2019 г.

Принято к публикации 15 апреля 2019 г.

\begin{abstract}
Методами атомно-силовой и магнитно-силовой микроскопии исследованы массивы пермаллоевых частиц с конфигурационной анизотропией формы, созданные с помощью микросферной литографии. Рассмотрено влияние размеров сферических частиц, используемых в качестве литографической маски, на распределение намагниченности в полученных частицах. Проведено компьютерное моделирование магнитно-силовых изображений частиц. Получены количественные оценки и сделано сравнение воспроизводимости формы частиц, полученных методом микросферой и сканирующей зондовой литографии.
\end{abstract}

Ключевые слова: магнитные частицы, литография, микромагнетизм, магнитно-силовая микроскопия.

DOI: 10.21883/JTF.2019.11.48338.121-19

\section{Введение}

Ферромагнитные частицы с симметричной геометрией (треугольные, квадратные и т.п.), обладающие конфигурационной анизотропией, могут иметь несколько квазиоднородных („near-uniform“) магнитных состояний [1-4], разделенных достаточно высокими энергетическими барьерами, что обеспечивает стабильность этих состояний во времени. Данный факт позволяет рассматривать такие частицы в качестве перспективных элементов для создания запоминающих магнитоэлектрических стрейнтронных ячеек памяти произвольного доступа (MeRAM Magnetoelectric Random Access Memory $[5,6])$. Ячейка MeRAM, состоящая из таких частиц, может хранить не один, а несколько бит информации [1]. В работах [1-4] авторами рассматривались планарные частицы пермаллоя (Ру) квадратной и треугольной формы с размерами сторон фигур от 50 до $500 \mathrm{~nm}$. Все частицы независимо от формы имели квазиоднородную намагниченность. Позднее нами были исследованы частицы Ру треугольной формы с разной степенью прогиба сторон треугольника [7]. Несмотря на бо́льшие, чем у авторов предыдущих работ, размеры (длина описанного равностороннего треугольника составляла $1.35 \mu \mathrm{m}$ ), частицы с вогнутыми сторонами находились в квазиоднородных состояниях, в то время как наиболее близкие по форме к равностороннему треугольнику частицы (без прогиба сторон) имели вихревую структуру намагниченности в отличие от равносторонних треугольных частиц с меньшими размерами сторон, рассмотренными в [2].

Ранее для получения частиц с конфигурационной анизотропией нами использовалась сканирующая зондовая литография (СЗЛ) [7,8]. К чувствительным недостаткам такого метода можно отнести слабую повторяемость частиц, что, в свою очередь, приводит к большой дисперсии значений поля переключения из одного квазиоднородного состояния в другое [7]. Один из перспективных способов создания хорошо повторяемых микрочастиц состоит в использовании одинаковых микросфер для литографического процесса. Суспензия микросфер определенной концентрации, наносимая на специально подготовленную подложку, за счет самосборки может выстроиться в регулярный плотноупакованный монослой, который служит литографической маской $[9,10]$.

Целью настоящей работы является установление зависимости распределения намагниченности Ру частиц от размеров сфер, используемых при литографии, в частности, определение условий, при которых в частицах треугольной формы за счет прогиба сторон формируется квазиоднородная намагниченность, а также сравнение преимуществ и недостатков методов СЗЛ и микросферной литографии для получения частиц одинаковой формы с минимальным отклонением от заданного среднего радиуса кривизны сторон треугольника.

\section{Методики приготовления образцов и проведения измерений}

Исследуемые в работе частицы Ру создавались методом микросферной литографии на стеклянной гидрофильной пластине размерами $14 \times 4 \mathrm{~mm}$ и толщиной $0.15 \mathrm{~mm}$. Для этого на нее наносилась капля $2.5 \%$ водной суспензии, содержащей сферы $\mathrm{SiO}_{2}$. После высыхания на подложке формировалась маска в виде монослоя 


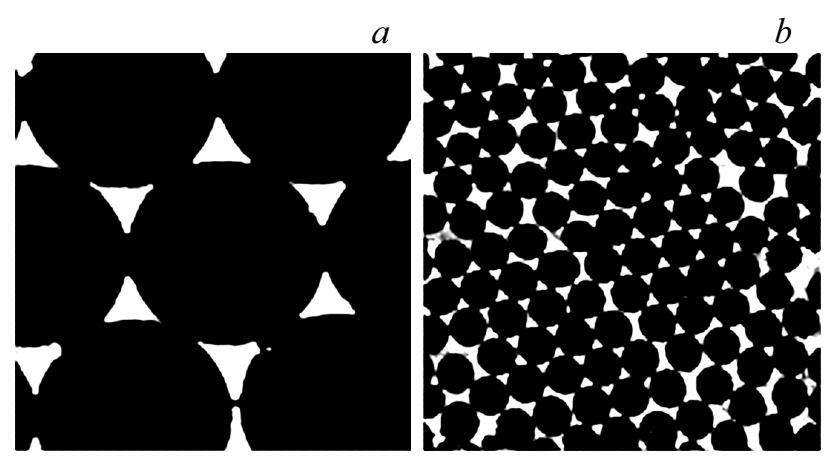

Рис. 1. АСМ изображения частиц, полученных с помощью микросферной литографии при плотной упаковке сфер в слое $(a)$, и при нарушениях в упаковке сфер $(b)$. Размер области сканирования $10 \times 10(a)$ и $49 \times 49 \mu \mathrm{m}(b)$, высота частиц $40 \mathrm{~nm}$.

сфер. Всего было изготовлено три образца с литографическими масками из суспензий с различным средним размером микросфер: $0.5,2.35$ и $5 \mu \mathrm{m}$ (с относительным стандартным отклонением от среднего размера $\pm 3 \%$ ).

Для нанесения слоя Ру (сплав 79НМ, основные компоненты: Ni 79\%, Fe 16\%, Мо 4\%) использовалась методика распыления электронным лучом твердотельной мишени. Напыление проводилось в условиях сверхвысокого вакуума на установке Omicron Multiprobe Р. Излишки металла и микросферы удалялись промыванием в изопропиловом спирте в ультразвуковой ванне. Далее образец промывался дистиллированной водой и высушивался. Сформированные частицы имели одинаковую высоту $40 \mathrm{~nm}$ и представляли собой треугольники с вогнутыми сторонами (рис. $1, a$ ). Частицы, полученные методом микросферной литографии, сравнивались с ранее изготовленными методом СЗЛ частицами [7] для количественной оценки воспроизводимости формы.

Размеры и форма частиц определялась с помощью сканирующего зондового микроскопа (С3М) Solver P47 Pro, работающего в режиме атомно-силовой микроскопии (АСМ). Для АСМ измерений использовался кантилевер D160 (SCDprobes) с алмазным пирамидальным монокристаллом, закрепленным на кремниевой балке. Распределение намагниченности в частицах исследовалось с помощью С3M Solver P47 Pro, paботающего в режиме магнитно-силовой микроскопии $(\mathrm{MCM})$. Для МСМ измерений использовались кантилеверы Multi75M-G (BudgetSensor). MCM измерения проводились в однопроходном режиме, при которой МСМ зонд перемещается над поверхностью образца на достаточном удалении (несколько десятков нанометров) для того, чтобы исключить перемагничивание частиц магнитным полем зонда.

Для определения распределения намагниченности в частице, исходя из полученного МСМ изображения, использовался следующий подход. Возможное распределение намагниченности в частице моделировалось при помощи программы OOMMF [11]. При этом использовалось ее трехмерное изображение, полученное в АСМ измерениях. Затем по рассчитанному распределению намагниченности моделировалось виртуальное МСМ изображение частицы при помощи разработанной ранее программы „Виртуальный микроскоп“ [12]. Полученное изображение сравнивалось с экспериментальным. При их совпадении делался вывод о том, что рассчитанное распределение намагниченности соответствует реальному. При ООММF-моделировании частица разбивалась на элементарные кубические ячейки с размером грани $5 \mathrm{~nm}$. Намагниченность насыщения пермаллоя принималась равной $750 \mathrm{kA} / \mathrm{m}$ [13]. Поскольку исследуемые частицы имели поликристаллическую структуру, константа магнитной кристаллической анизотропии принималась равной нулю.

\section{АСМ пермаллоевых частиц, полученных при помощи различных литографических масок}

Анализ АСМ изображений больших площадей подложки с частицами показал, что не всегда удается получить плотноупакованный монослой сфер, поэтому на поверхности могут встречаться и частицы произвольной формы (рис. $1, b)$. АСМ изображения частиц, полученных микросферной литографией с использованием сфер диаметром $0.5,2.35$ и $5 \mu$ m, представлены на рис. $2, a-c$. Для сравнения на рис. $2, d$ показано АСМ изображение частицы, изготовленной методом СЗЛ.

ACM измерения Ру частиц, изготовленных при помощи маски, состоящей из сфер диаметром $0.5 \mu \mathrm{m}$, показали, что по форме они близки к равностороннему треугольнику с прямыми сторонами. В этом случае радиус закругления стороны треугольника стремится к бесконечности. Несмотря на полученные с помощью данных сфер сравнительно одинаковые частицы, они не могут корректно сравниваться с частицами, обладающими конечным радиусом закругления, и поэтому далее не будут рассматриваться для оценки формы.

Согласно [2], величина поля анизотропии (которое в случае однородно намагниченных структур совпадает с полем переключения) и энергии анизотропии плоской частицы (латеральные размеры значительно больше высоты) прямо пропорциональна корню квадратному из ее площади. Поэтому представляется интересным количественно оценить среднеквадратическое отклонение от среднего значения площади для частиц с конфигурационной анизотропией, изготовленных с помощью различных литографических масок. Для корректного сравнения площадей частиц $(S)$ каждое полученное абсолютное значение нормировалось на площадь равностороннего треугольника $\left(S_{\mathrm{tr}}\right)$, описанного вокруг частицы. Средние значения приведенных площадей частиц $\left(\left\langle S / S_{\text {tr }}\right\rangle\right)$ и среднеквадратические отклонения $(\sigma)$ приведены в табл. 1 , из которой видно, что приведенная площадь частиц, изготовленных при помощи различных масок, примерно одинакова. 
Еще одним фактором, существенно влияющем на величину поля анизотропии, является наличие дефектов. В широком смысле можно трактовать вогнутость сторон частиц как ,дефект“ - отклонение от формы равностороннего треугольника. Поэтому интересным представляется измерить радиус закругления $(R)$ всех трех сторон частиц, изготовленных при помощи разных масок. В идеальных частицах все три радиуса должны быть идентичными, кроме того, очевидно, что в частицах, изготовленных методом микросферной литографии, радиус закругления должен совпадать с радиусом микросферы. Различие в форме частиц, изготовленных микросферной литографией, обусловлены в первую очередь наличием в суспензии микросфер разного диаметра (относительная ошибка - 3\% по паспортным данным). Еще одним фактором может стать неплотное прилегание микросфер к подложке или их осаждение на подложку в несколько слоев, что может приводить не только к различию в размерах частиц, но и к изменению их формы. Частицы, изготовленные СЗЛ, отличаются по форме и размерам в основном из-за неравномерного удаления зондом полимера от точки к точке во время создания маски. Более подробно методы СЗЛ описаны в работе [8]. Результаты измерения радиусов закругления сторон треугольных частиц $(R)$, изготовленных при помощи различных литографических масок, представлены в табл. 2.

Из сравнения величин $\delta$ (отношение среднеквадратического отклонения к среднему значению радиуса
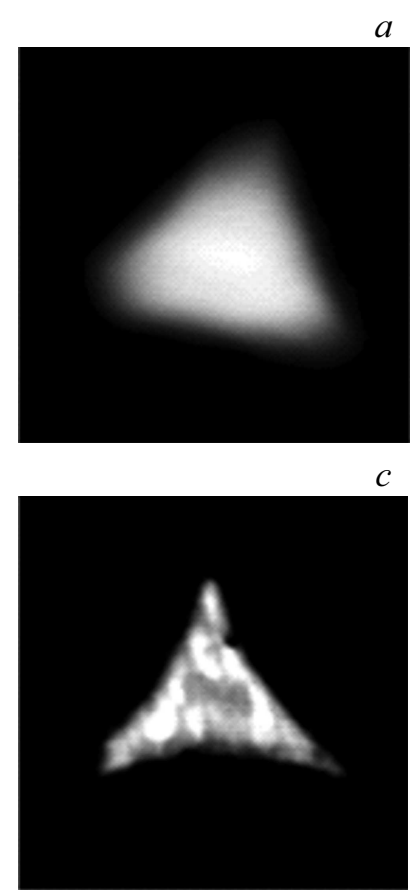

\begin{abstract}
$c$
\end{abstract}
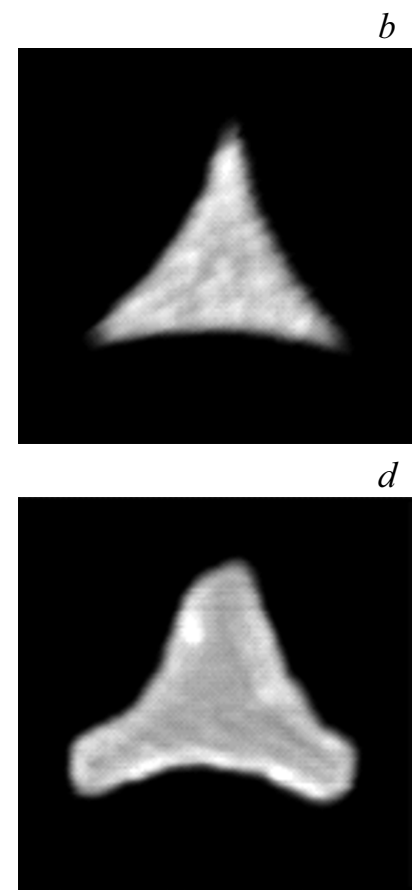

Рис. 2. АСМ изображение частицы, полученной с помощью микросферной литографии с использованием сфер диаметром $0.5(a), 2.35(b), 5 \mu \mathrm{m}(c)$, высота частиц $40 \mathrm{~nm}$. АСМ изображение частицы, полученной с помощью СЗЛ $(d)$, высота частицы $30 \mathrm{~nm}$. Размер области сканирования для рисунка: $a-380 \times 380 \mathrm{~nm}, b-1.21 \times 1.21, c-2.9 \times 2.9, d-$ $1.75 \times 1.75 \mu \mathrm{m}$.
Таблица 1. Средняя приведенная площадь частиц $\left\langle S / S_{\mathrm{tr}}\right\rangle$

\begin{tabular}{c|c|c}
\hline Диаметр сферы $2.35 \mu \mathrm{m}$ & Диаметр сферы $5 \mu \mathrm{m}$ & \multicolumn{1}{c}{ СЗЛ } \\
\hline$\left\langle S / S_{\text {tr }}\right\rangle=0.88 ;$ & $\left\langle S / S_{\text {tr }}\right\rangle=0.74 ;$ & $\left\langle S / S_{\text {tr }}\right\rangle=0.83$ \\
$\sigma=0.05$ & $\sigma=0.04$ & $\sigma=0.03$
\end{tabular}

Таблица 2. Средний радиус закругления стороны частицы с конфигурационной анизотропией $\langle R\rangle$, среднеквадратическое отклонение $(\sigma)$ и отношение среднеквадратического отклонения к среднему значению радиуса закругления $(\delta)$

\begin{tabular}{c|c|c}
\hline Диаметр сферы $2.35 \mu \mathrm{m}$ & Диаметр сферы $5 \mu \mathrm{m}$ & С3Л \\
\hline$\langle R\rangle=1.1 \mu \mathrm{m}$ & $\langle R\rangle=2.2 \mu \mathrm{m}$ & $\langle R\rangle=0.87 \mu \mathrm{m}$ \\
$\sigma=0.09 \mu \mathrm{m}$ & $\sigma=0.2 \mu \mathrm{m}$ & $\sigma=0.168 \mu \mathrm{m}$ \\
$\delta=8.3 \%$ & $\delta=9.2 \%$ & $\delta=19.4 \%$
\end{tabular}

закругления) следует, что частицы, полученные методом СЗЛ, имеют существенно меньшую воспроизводимость формы в сравнении с частицами, полученными методом микросферной литографии.

\section{МСМ пермаллоевых частиц, полученных методом микросферной литографии}

Наиболее перспективными для создания ячеек MeRAM являются частицы с однородной или квазиоднородной намагниченностью, имеющие минимальные размеры $[1-4,6]$. Для создания таких частиц необходимо использовать микросферы с минимальным диаметром. Однако в этом случае также снижается и расстояние между частицами, что может приводить к их взаимодействию друг с другом. В нашем случае при создании частиц минимальный диаметр используемых микросфер составил $0.5 \mu \mathrm{m}$, при этом среднее расстояние между полученными частицами составило $0.1 \mu \mathrm{m}$, а средняя длина стороны частицы - $0.2 \mu \mathrm{m}$. Расстояние между частицами принималось равным расстоянию от вершины частицы до ближайшей вершины другой частицы. АСМ изображение частицы представлено на рис. 2,a. МСМ исследования показали, что такие частицы обладают квазиоднородной намагниченностью, что совпадает с результатом, полученным в работе [2]. МСМ изображение частиц представлено на рис. 3,a. Однако из-за малого расстояния между частицами, для получения магнитного изображения от отдельных частиц зонд должен сильно приблизиться к поверхности, иначе МСМ изображение будет представлять результат взаимодействия зонда сразу с несколькими частицами. Уменьшение расстояния зонд-образец приводит к увеличению влияния магнитного зонда на исследуемую частицу и в некоторых случаях [7] - к изменению ориентации квазиоднородной намагниченности такой частицы. Кроме того, моделирование показало наличие существенного магнитостатического взаимодействия между соседними частицами. 


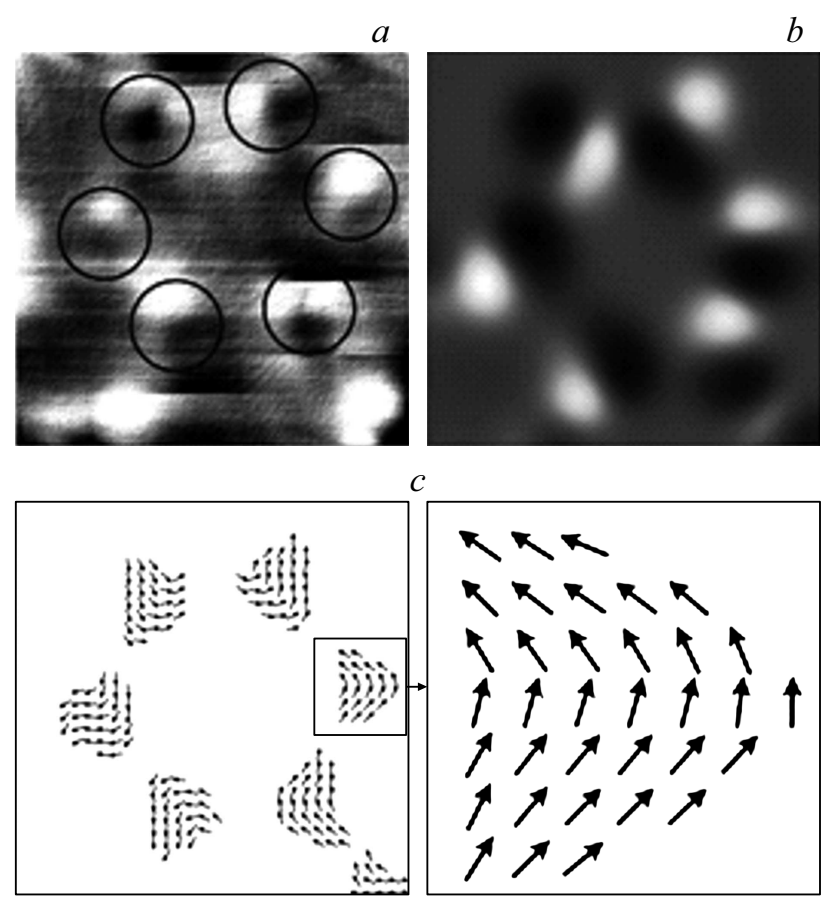

Рис. 3. МСМ изображение частиц высотой $40 \mathrm{~nm}$, полученных с помощью микросферной литографии с использованием сфер $0.5 \mu \mathrm{m}(a)$. Виртуальное МСМ изображение $(b)$, полученное на основе распределения локальной намагниченности в частицах $(c)$, смоделированного при помощи ООММF. Размер области сканирования $1.1 \times 1.1 \mu \mathrm{m}$.

При использовании микросфер бо́льшего диаметра увеличивается как расстояние между частицами, так и их размер. Это позволяет получать МСМ изображения без риска перемагничивания исследуемых структур зондом. На рис. 2, $b$ приведено АСМ изображение частицы, полученной с помощью сфер диаметром $2.35 \mu \mathrm{m}$. Среднее расстояние между частицами в этом случае составляет $0.35 \mu \mathrm{m}$, а средний размер стороны частицы $-0.85 \mu \mathrm{m}$. МСМ измерения показали, что эти частицы также обладают квазиоднородной намагниченностью (рис. $4, b$ ).

При использовании микросфер диаметром $5 \mu \mathrm{m}$ среднее расстояние между частицами составляет $0.74 \mu \mathrm{m}$, средний размер стороны равен $1.73 \mu \mathrm{m}$ (рис. 1, $a, 2, c)$. МСМ исследования показали, что частицы такого размера теряют квазиоднородную намагниченность (рис. 5, $b$ ).

Для расшифровки МСМ изображений было проведено моделирование распределения намагниченности в массиве частиц с помощью программы ООММF. Смоделированные МСМ изображения и распределения намагниченности приведены для каждой ситуации на рис. 3-5, b, c. Согласно полученным данным, квазиоднородная намагниченность сохраняется в частицах, полученных микросферной литографией с диаметром сфер 0.5 и $2.35 \mu \mathrm{m}$, что хорошо согласуется с экспериментом.

Проведенные ранее эксперименты с треугольными частицами с вогнутыми сторонами, полученными с помощью СЗЛ [7], показали, что они находятся в казиодно- родном состоянии намагниченности. Исходя из табл. 2, частицы, изготовленные методом СЗЛ со средним размером стороны, равным $1.35 \mu \mathrm{m}$, имеют бо́льший прогиб, чем изготовленные с помощью микросферной литографии. Согласно [2], это увеличивает поле анизотропии частицы и, как следствие, усиливает стабильность такого квазиоднородного состояния. Поэтому данные частицы находятся в таком состоянии, несмотря на то, что по линейным размерам сторон они были близки к частицам, изготовленным с помощью $5 \mu \mathrm{m}$ сфер, которые имеют вихревую структуру намагниченности (рис. 5, c).

По результатам проведенных исследований к преимуществам микросферной литографии по сравнению с СЗЛ можно отнести:

- более быстрый процесс изготовления литографической маски. Нанесение капли суспензии на подложку и процесс высыхания занимает не более $15 \mathrm{~min}$. В СЗЛ необходимо несколько стадий: нанесение полимера на подложку, сушка при повышенной температуре и собственно процесс изготовления маски в СЗМ по шаблону, который требует нескольких часов работы;

- большую активную площадь, заполненную частицами, область заполнения сравнима с размером нанесенной капли суспензии. СЗЛ ограничена размерами пьезосканера (порядка $100 \mu \mathrm{m}$ и менее);

- высокую воспроизводимость формы частиц (табл. 2) из-за использования одинаковых сфер с малым отклонением диаметра от среднего значения. В СЗЛ получить одинаковые частицы сложной формы (например,
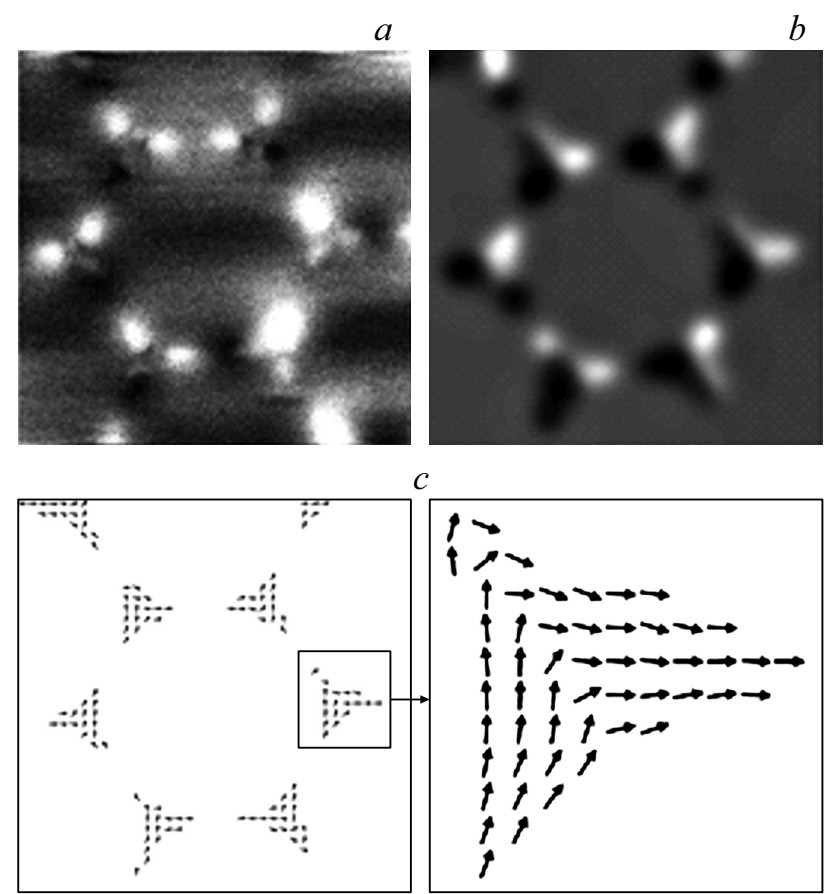

Рис. 4. МСМ изображение частиц высотой $40 \mathrm{~nm}$, полученных с помощью микросферной литографии с использованием сфер $2.35 \mu \mathrm{m}(a)$. Виртуальное МСМ изображение $(b)$, полученное на основе распределения локальной намагниченности в частицах $(c)$, смоделированного при помощи ООММF. Размер области сканирования $4.6 \times 4.6 \mu \mathrm{m}$. 

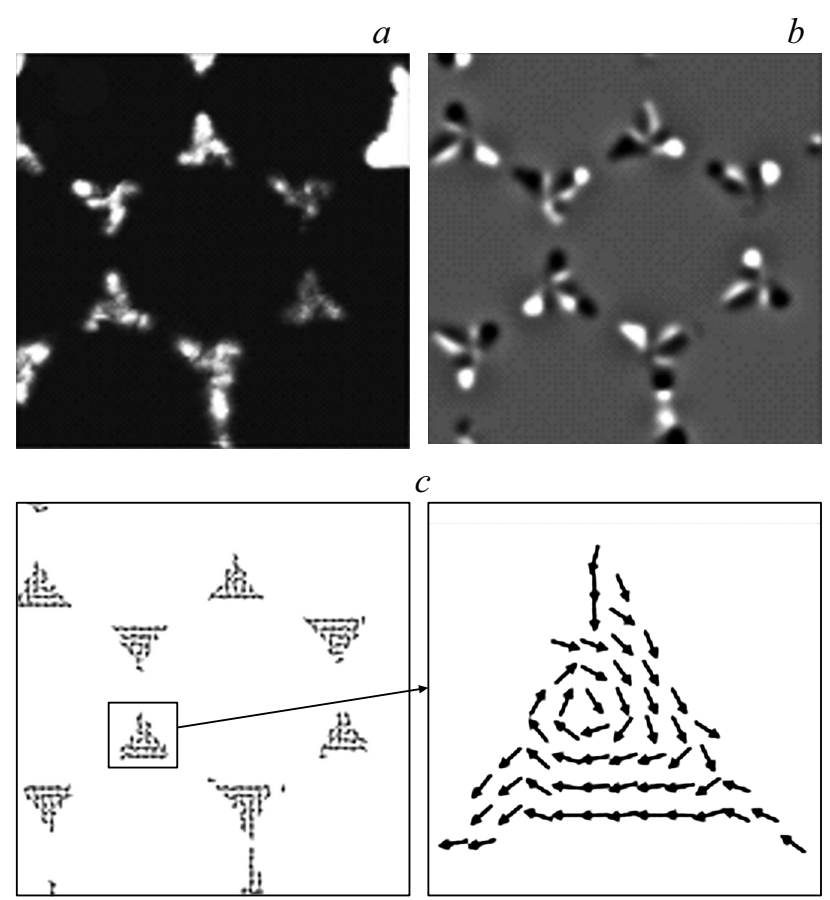

$c$

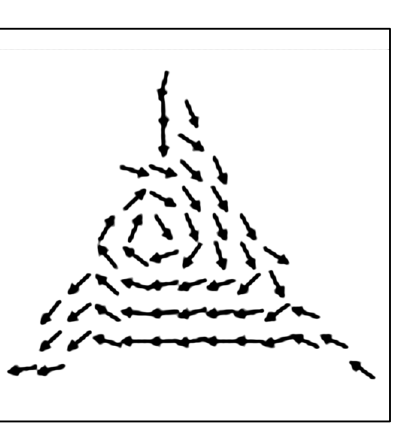

Рис. 5. МСМ изображение частиц высотой $40 \mathrm{~nm}$, полученных с помощью микросферной литографии с использованием сфер $5 \mu \mathrm{m}(a)$. Виртуальное МСМ изображение $(b)$, полученное на основе распределения локальной намагниченности в частицах $(c)$, смоделированного при помощи ООММF. Размер области сканирования $10 \times 10 \mu \mathrm{m}$.

частицы с конфигурационной анизотропией) затруднительно из-за деградации зондов, растрового (поточечного) процесса литографии с конечным шагом, возможного наличия термодрейфа пьезосканера.

К недостаткам микросферной литографии относятся: - относительно высокая сложность подготовки исходных материалов: гидрофильная подложка, точный подбор концентрации суспензии, чистота растворителей и т. п.;

- случайный характер расположения частиц на подложке; расстояние между частицами, их размеры и форма фиксированы для выбранного размера сфер, что приводит, например, к возникновению магнитостатического взаимодействия между частицами, полученными литографией микросферами диаметром $500 \mathrm{~nm}$.

- невозможность повторной литографии тем же методом (например, для нанесения второго слоя).

\section{Заключение}

Таким образом, продемонстрировано, что литография масками, изготовленными из слоя микросфер, подходит для получения больших массивов частиц, размеры и геометрические параметры которых существенно стабильнее, чем у частиц, изготавливаемых СЗЛ. Полученные таким образом частицы обладают конфигурационной анизотропией, которая может приводить к появлению квазиоднородной намагниченности. Такое магнитное со- стояние сохраняется в частицах, полученных с помощью микросферной литографии с диаметром сфер до $2.35 \mu \mathrm{m}$. При использовании микросфер диаметром $5 \mu \mathrm{m}$ состояние квазиоднородной намагниченности теряется, и образуется вихревая структура.

\section{Финансирование работы}

Работа выполнялась в рамках Государственных заданий Федерального исследовательского центра „Казанский научный центр РАН“ и Института физики твердого тела РАН. МСМ исследования проводились при поддержке РФФИ проект 18-02-00204.

\section{Конфликт интересов}

Авторы заявляют, что у них нет конфликта интересов.

\section{Список литературы}

[1] Salehi-Fashami M., D’Souza N. // JMMM. 2017. Vol. 438. P. 76-84. DOI: 10.1016/j.jmmm.2017.02.003

[2] Nanayakkara K., Vasil'evskii I.S., Eremin I.S., Kolentsova O.S., Kargin N.I., Anferov A., Kozhanov A. // J. Appl. Phys. 2016. Vol. 119. P. 233906. DOI: 10.1063/1.4954162

[3] Lambson B., Gu Z., Monroe M., Dhuey S., Scholl A., Bokor J. // Appl. Phys. A. 2013. Vol. 111. P. 413-421. DOI: 10.1007/s00339-013-7654-y

[4] Koltsov D., Welland M. // J. Appl. Phys. 2003. Vol. 94. P. 3457. DOI: $10.1063 / 1.1595705$

[5] Atulasimha J., Bandyopadhyay S. Nanomagnetic and Spintronic Devices for Energy-Efficient Memory and Computing. Chichester: WILEY, 2016. 352 p.

[6] Бухараев А.А., Звездин А.К., Пятаков А.П., Фетисов Ю.К. // УФН. 2018. Т. 188. Вып. 12. С. 1288-1330. DOI: 10.3367/UFNr.2018.01.038279 [Bukharaev A.A., Zvezdin A.K., Pyatakov A.P., Fetisov Yu.K. // Phys. Usp. 2018. Vol. 61. P. 1175-1212. DOI: 10.3367/UFNe.2018.01.038279]

[7] Bizyaev D.A., Bukharaev A.A., Chuklanov A.P., Nurgazizov N.I. // Phys. Solid State. 2018. Vol. 60. N 11. P. 2194-2199. DOI: $10.1134 / \mathrm{S} 1063783418110021$

[8] Бизяев Д.А., Бухараев А.А., Зиганшина С.А., Нургазизов Н.И., Ханипов Т.Ф., Чукланов А.П. // Микроэлектроника. 2015. Т. 44. № 6. С. 437-447. DOI: $10.7868 / \mathrm{S} 054412691505004 \mathrm{X}$ [Bizyaev D.A., Bukharaev A.A., Ziganshina S.A., Nurgazizov N.I., Khanipov T.F., Chuklanov A.P. // Russian Microelectronics. 2015. Vol. 44. N 6. P. 389-398. DOI: 10.1134/S1063739715050042]

[9] Zhong H., Tarrach G., Wu P., Drechsler A., Wei D., Yuan J. // Nanotechnology. 2008. Vol. 19. N 9. P. 095703. DOI: $10.1088 / 0957-4484 / 19 / 9 / 095703$

[10] Zhang Z., Geng C., Hao Zh., Wei T., Yan Q. // Adv. Colloid Interface Sci. 2016. Vol. 228. P. 105-122. DOI: $10.1016 /$ j.cis.2015.11.012

[11] Donahue-M.J., Porter-D.G. OOMMF. http://math.nist.gov/oommf/

[12] Овчинников Д.В., Бухараев А.А. // ЖТФ. 2001. Т. 71. Вып. 8. C. 85-91. [Ovchinnikov D.V., Bukharaev A.A. // Tech. Phys. 2001. Vol. 46. N 8. P. 1014-1019. DOI: https://doi.org/10.1134/1.1395123]

[13] Кикоин И.К. Таблицы физических величин. Справочник. М.: Атомиздат, 1976. 1008 с. 